\title{
EVALUATION OF THE EFFECTIVENESS OF SELECTED TECHNIQUES FOR ENDOTRACHEAL INTUBATION IN CONDITIONS OF SIMULATED RESUSCITATION PERFORMED BY NURSES
}

\author{
Domagoj Buljan ${ }^{1}$, Joanna Rosiczuk², Iwona Stawicka ${ }^{3}$ \\ 1 University of J.J. Strossmayer Osijek, Osijek, Croatia \\ ${ }^{2}$ Department of Nervous System Diseases, Faculty of Health Science, Wroclaw Medical University, Wroclaw, Poland \\ ${ }^{3}$ Polish Society of Disaster Medicine, Poland
}

Sir,

During cardiac arrest, the oxygen reserve in the body in normothermia conditions is sufficient for about 3-5 minutes, after which time there are irreversible changes in the vital organs, in particular those sensitive to hypoxia in the central nervous system. Therefore, clearing and protecting the airways, as well as the administration of oxygen, are key elements in resuscitation. According to the current guidelines of the European Resuscitation Council (ERC) and the American Heart Association (AHA), the gold standard for airway management during cardiopulmonary resuscitation (CPR) is endotracheal intubation. These guidelines indicate that in order to minimize interruptions in chest compressions, intubation should be carried out during chest compressions. Studies show that patient movements as a result of CC cause a decrease in the efficiency of intubation using direct laryngoscopy [1]. An additional difficulty may be swelling of the tongue. In such conditions, intubation may be difficult or completely impossible to carry out [2,3]. The search for alternative methods of intubation is, therefore, an important issue.

The aim of the study was to compare the intubation time and the efficiency of endotracheal intubation in the case of an adult with the use of two types of laryngoscope under simulated CPR performed by nurses.

The study involved 47 nurses. We studied 39 women $(82.9 \%)$ and 8 men (17.1\%), aged 24 to
41 years (mean 34.5 years). In order to simulate a patient requiring intubation, a SimMan 3G (Laerdal, Norway) manikin was used. In order to simulate CPR, LifeLine $\mathrm{ARM}^{\circledR}$ (Defibtech, USA) modern mechanical chest compression devices were used in asynchronous mode, as studies show medical personnel perform manual CPR at a low level of efficiency [4, 5], which could reduce the efficacy results for intubation. Difficult airway was obtained by causing edema tongue in the manikin control panel. Thus, a level of 3 according to the Cormack-Lehane scale was observed. The level of difficulty was confirmed by a doctor specialized in anesthesia and intensive care. The participants in the study performed endotracheal intubation using four different devices for intubation: 1) Laryngoscope with a "gold standard" Macintosh with a size 3 blade (MAC Mercury Medical, Clearwater, USA); 2) TruView PCD EVO2 Laryngoscope with a size 3 blade (PCD; CD; Truphatek Holdings, Ltd., Netanya, Israel) used as a video laryngoscope (Figure 1). In all intubation cases, an endotracheal tube of size ID 7.0 was used. In the case of PCD, a special semi-rigid stylet was employed, while in the case of MAC we used a standard semi-rigid stylet. Before the study, all the participants took part in a 60-minute training session in the anatomy, physiology and pathophysiology of respiration, which also included various techniques for airway control. After the theoretical part and a demonstration of the proper endotracheal intubation technique with the 


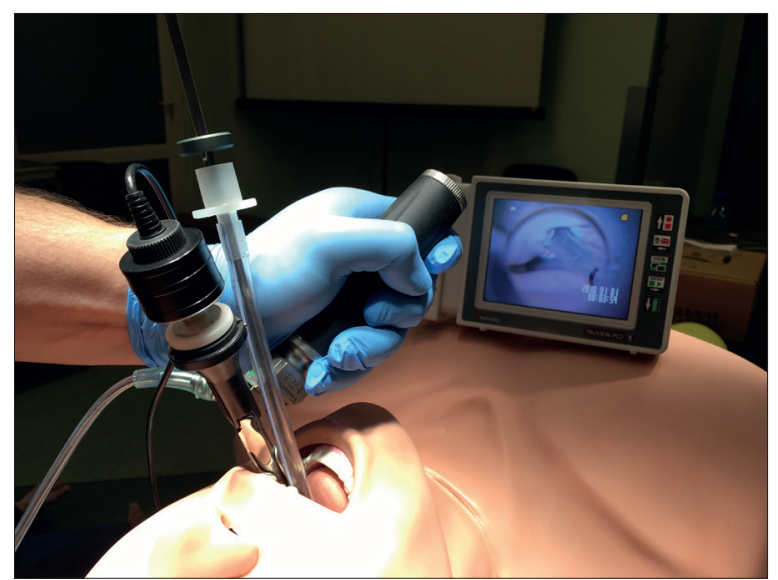

FIGURE 1. Truview EVO2 PCD videolaryngoscope

use of the appliances concerned, the participants took part in a training session during which they performed endotracheal intubation using a MAC and a PCD. The session lasted until each participant had correctly performed three intubations with each device. Using Research Randomizer (www. randomizer.org), the participants in the study were randomly assigned to one of the two devices. Both the order of participants and the order of device use were random.

In the case of the PCD, the obtained time to visualize the larynx was $13.5 \mathrm{~s}(12.5-16)$. while the time for the MAC was longer at 16.5s (14-22) $(p=0.003)$. The longest time for the insertion of the tube through the vocal cords was observed for intubation using a MAC [41.5 s (35-45)] while in the case of the PCD this was 29s $(24-32.5)(p<0.001)$. The time of the first ventilation sample for emergency resuscitation in difficult airways in the case of the MAC was $53.5 \mathrm{~s}$ (42-57.5), which was significantly longer than in the case of the PCD, at $37 \mathrm{~s}(33-39,5)$ $(p<0.001)$.

The effectiveness of the first attempt of the MAC intubation was $29.8 \%$, which was significantly lower than that for the PCD $(70.2 \% ; p<0.001)$. With the $P C D$, overall intubation efficacy was $82.9 \%$, while in the case of the MAC this was $38.3 \%$.

In conclusion, the study showed that the efficacy of endotracheal intubation performed by nurses is insufficient. After minimal training, however, nurses are able to perform intubation with a high level of efficiency using videolaryngoscopes.

\section{REFERENCES}

1. Liu Y, Xue FS, Cheng Y. Endotracheal intubation with the Pentax-Airwayscope and Macintosh laryngoscope during chest compression. Eur J Emerg Med, 2013; 20(2): 142-144. doi: 10.1097/MEJ. 0b013e3283573e44.

2. Bogdański Ł, Truszewski Z, Kurowski A et al. Simulated endotracheal intubation of a patient with cervical spine immobilization during resuscitation: a randomized comparison of the Pentax AWS, the Airtraq, and the McCoy Laryngoscopes. Am J Emerg Med, 2015; 33(12): 1814-1817. doi: 10.1016/j.ajem.2015.09.005.

3. Farag E. Airway management for cervical spine surgery. Best Pract Res Clin Anaesthesiol, 2016; 30(1): 13-25. doi: 10.1016/ j.bpa.2016.01.001.

4. Szarpak $Ł$, Truszewski Z, Smereka J, Czyżewski Ł. Does the use of a chest compression system in children improve the effectiveness of chest compressions? A randomized crossover simulation pilot study. Kardiol Pol, 2016 Jul 8. doi: 10.5603/KP.a2016.0107.

5. Kurowski A, Szarpak Ł, Bogdanski L. Comparison of the effectiveness of cardiopulmonary resuscitation with standard manual chest compressions and the use of TrueCPR and PocketCPR feedback devices. Kardiol Pol, 2015; 73(10): 924-930. doi: 10.5603/KP.a2015.0084. 